\title{
A Method for Solving the Three-dimensional Wave Equation
}

\author{
A. S. Yakimov \\ Tomsk State University, Tomsk, 634034, Russia \\ *Corresponding Author: yakimovas@mail.ru
}

Copyright $@ 2013$ Horizon Research Publishing All rights reserved.

\begin{abstract}
On the basis of the Laplace integral transform, locally one-dimensional scheme of cleavage and quasi-linearization method to obtain an approximate analytical solution of the three-dimensional nonlinear hyperbolic equation of second order. The assessment of the accuracy of analytical formulas when compared with the exact solution of the first boundary value problem and numerical solution by a known method.
\end{abstract}

Keywords Analytical Solution, Three-dimensional Telegraph Equation, Laplace Integral Transform

\section{Introduction}

In mathematical modeling of heat and mass transfer [1], the heat transfer in high-frequency processes [2], vibrations [3] and so on. there is a problem the solution of telegraph type an equation [1-3]. If the solution of a nonlinear parabolic equation [1] there are a number of analytical techniques (reviewed in [4]), the exact analytical solutions are obtained for the linear one-dimensional (in the absence of a source) [5,6] or multi-dimensional [3] an equation of the telegraph type. However, in practice most often of interest to the solution of nonlinear boundary-value problems [1-4,7].

For one-dimensional solutions of nonlinear ordinary differential equations in [8] proposed a method of quasi-linearization. With this method, a decision along the nonlinear problem is reduced to solving a sequence of linear problems, which is essentially a development of the well-known Newton's method and its generalized variant proposed by L. V. Kantorovich [9]. Otherwise, the quasi-linearization - is the application of a nonlinear functional generated by the nonlinear boundary value problem, the Newton-Kantorovich.

In the numerical solution of problems of mathematical physics were effective splitting methods [10,11]. In particular, the locally one-dimensional scheme cleavage [10] proposed to solve the multi-dimensional heat equation in combination with the analytical (constant coefficients) and numerical methods

The purpose of article - with the help of the locally one-dimensional scheme splitting [11], quasi-linearization $[8,9]$ and the Laplace integral transform [12] to find an approximate analytical solution of the nonlinear three-dimensional hyperbolic heat conduction equation in a finite region and to assess the accuracy of analytical formulas.

\section{Statement of the Problem and the Algorithm of Method}

Suppose you want to find a solution to a hyperbolic equation of the second order [1,3], with sources

$$
\begin{gathered}
z^{-1} \frac{\partial^{2} T}{\partial t^{2}}+C(T) \frac{\partial T}{\partial t}= \\
\sum_{j=1}^{3}\left\{\frac{\partial}{\partial x_{j}}\left[A(T) \frac{\partial T}{\partial x_{j}}\right]+Y_{j}(T) \frac{\partial T}{\partial x_{j}}\right\}+A_{1} T^{k}+A_{2}(x, t)
\end{gathered}
$$

in the parallelepiped $Q:\left[x=\left(x_{1}, x_{2}, x_{3}\right), 0<x_{j}<L_{j}\right.$, $\left.0<L_{j}<\infty, j=1,2,3\right], \bar{Q}=Q+\Gamma, \bar{Q}_{t}=\bar{Q} \times\left[0<t \leq t_{0}\right]$, $\Gamma$ - boundary surface of the domain $Q$ with initial conditions

$$
\left.T\right|_{t=0}=p_{1}(x),\left.\frac{\partial T}{\partial t}\right|_{t=0}=p_{2}(x), x=\left(x_{1}, x_{2}, x_{3}\right)
$$

and to simplify further calculations with the boundary condition of the first kind

$$
\left.T\right|_{\Gamma}=\Psi, \quad \Psi \neq \text { const }
$$

where $A_{1}=$ const, $z=c^{2} / A_{\mathrm{H}}, Y_{j}=C(T) w_{j}(T), j=$ $1,2,3, c$ - the speed of propagation of thermal perturbations (speed of sound in the medium), $\mathrm{m} / \mathrm{s} ; w_{j}(T), j=1,2,3-$ the rate of convective heat transfer, $\mathrm{m} / \mathrm{s} ; C(T)-$ the coefficient of volumetric heat capacity, $\mathrm{J} /\left(\mathrm{m}^{3} \cdot \mathrm{K}\right) ;(A(T)$ 
- the thermal conductivity, $A_{\mathrm{H}}-$ the thermal conductivity at the initial temperature, $\mathrm{W} /(\mathrm{m} \cdot \mathrm{K}) ; T$ - the temperature, $\mathrm{K}$; $x_{j}, j=1,2,3$ - axis of the Descartes coordinate system, $\mathrm{m} ; t$ - time, $\mathrm{s} ; L_{j}, j=1,2,3$ - length of the sides of the parallelepiped, $\mathrm{m}$.

According to [1], the equation (1) is obtained assuming that $C(T), Y_{j}(T), j=1,2,3$ do not explicitly depend on time $t$ and $w_{j}<<1 \mathrm{~m} / \mathrm{s}, j=1,2,3$ disregard mixed derivative $\sum_{j=1}^{3} w_{j} \frac{\partial^{2} T}{\partial t \partial x_{j}}$ as compared $\partial^{2} T / \partial t^{2}$ to the left of equation (1). Furthermore, in equation (1) on the right side are absent form mixed derivatives $\sum_{j=1, j \neq i}^{3} \frac{\partial}{\partial x_{j}}\left(A(T) \frac{\partial T}{\partial x_{i}}\right)$ and summand $-\tau_{r}\left(\partial A_{2} / \partial t\right)$, $\tau_{r}=\chi / c^{2}\left(\tau_{r}\right.$ - relaxation time, $\chi$ - coefficient thermal diffusivity $\mathrm{m}^{2} / \mathrm{s}$ ), whose value for the times considered below: $t>100 \times \tau_{r}\left(\tau_{r} \approx 10^{-9} \mathrm{~s}\right)$ is negligible.

We will always assume:

1. Problem (1) - (3) has a unique solution $T(x, t)$, which continuously in $\bar{Q}_{t}$ and has continuous derivatives $\partial T / \partial t, \partial^{2} T / \partial t^{2}, \partial T / \partial x_{j}, \partial^{2} T / \partial x_{j}^{2} j=1,2,3.2$.

Performed following conditions: $A(T) \geq l_{1}>0$, $C(T) \geq l_{2}>0, z \geq l_{3}>0, l_{i}=$ const $, i=1,2,3$, $p_{1}, p_{2}$ - set to continuous function in $\bar{Q}$, a $C, A, Y_{j}$, $A_{2}, j=1,2,3$ - continuous functions in $\bar{Q}_{t}$. The coefficients $C(T), Y_{j}(T), j=1,2,3$ in the general case can be non-linearly dependent on the solution of problem [1]. 3. View $A(T)$ is defined by the following formula (16); $\Psi$ - given continuous function on the boundary $\Gamma$ for $0<t \leq t_{0}$, having bounded partial derivatives of the first order.

Applicable the locally one-dimensional scheme splitting of the equations (1) - (3) on the differential level [11] and introduce the superscripts (1), (2), (3) to denote the solution of the intermediate stages, as well as $\xi$ there is the direction of wave decision and $\eta$ there is direction the solution of the parabolic part equation (1). Then we have

$$
\begin{gathered}
z^{-1} \frac{\partial^{2} T_{\xi}^{(1)}}{\partial t^{2}}=\xi \frac{\partial}{\partial x_{1}}\left[A\left(T_{\xi}^{(0)}\right) \frac{\partial T_{\xi}^{(1)}}{\partial x_{1}}\right]+\xi \sigma_{1} A_{2}, \\
0<t<t_{*}, \\
\left.T_{\xi}^{(1)}\right|_{t=0}=p_{1}(x),\left.\frac{\partial T_{\xi}^{(1)}}{\partial t}\right|_{t=0}=p_{2}(x), 0<x_{j}<L_{j},
\end{gathered}
$$

$$
\begin{aligned}
& j=1,2,3, \\
& \left.T_{\xi}^{(1)}\right|_{x_{1}=0}=G_{1}\left(t_{*}, x_{2}, x_{3}\right) \text {, } \\
& \left.T_{\xi}^{(1)}\right|_{x_{1}=L_{1}}=G_{2}\left(t_{*}, L_{1}, x_{2}, x_{3}\right), \\
& C\left(T_{\xi}^{(1)}\right) \frac{\partial T_{\eta}^{(1)}}{\partial t}=\eta \frac{\partial}{\partial x_{1}}\left[A\left(T_{\xi}^{(1)}\right) \frac{\partial T_{\eta}^{(1)}}{\partial x_{1}}\right]+ \\
& Y_{1}\left(T_{\xi}^{(1)}\right) \frac{\partial T_{\eta}^{(1)}}{\partial x_{1}}+ \\
& +\sigma_{1}\left[A_{1}\left(T_{\eta}^{(1)}\right)^{k}+\eta A_{2}\right], 0<t<t_{*}, \\
& T_{\eta}^{(1)}(0, x)=T_{\xi}^{(1)}\left(t_{*}, x\right),\left.T_{\eta}^{(1)}\right|_{x_{1}=0}=G_{1}, \\
& \left.T_{\eta}^{(1)}\right|_{x_{1}=L_{1}}=G_{2} ; \\
& z^{-1} \frac{\partial^{2} T_{\xi}^{(2)}}{\partial t^{2}}=\xi \frac{\partial}{\partial x_{2}}\left[A\left(T_{\eta}^{(1)}\right) \frac{\partial T_{\xi}^{(2)}}{\partial x_{2}}\right]+\xi \sigma_{2} A_{2}, \\
& 0<t<t_{*}, \\
& T_{\xi}^{(2)}(0, x)=T_{\eta}^{(1)}\left(t_{*}, x\right), \frac{\partial T_{\xi}^{(2)}(0, x)}{\partial t}=\frac{\partial T_{\eta}^{(1)}\left(t_{*}, x\right)}{\partial t}, \\
& \left.T_{\xi}^{(2)}\right|_{x_{2}=0}=Q_{1}\left(t_{*}, x_{1}, x_{3}\right), \\
& \left.T_{\xi}^{(2)}\right|_{x_{2}=L_{2}}=Q_{2}\left(t_{*}, x_{1}, L_{2}, x_{3}\right), \\
& C\left(T_{\xi}^{(2)}\right) \frac{\partial T_{\eta}^{(2)}}{\partial t}=\eta \frac{\partial}{\partial x_{2}}\left[A\left(T_{\xi}^{(2)}\right) \frac{\partial T_{\eta}^{(2)}}{\partial x_{2}}\right]+ \\
& Y_{2}\left(T_{\xi}^{(2)}\right) \frac{\partial T_{\eta}^{(2)}}{\partial x_{2}}+ \\
& +\sigma_{2}\left[A_{1}\left(T_{\eta}^{(2)}\right)^{k}+\eta A_{2}\right], 0<t<t_{*}, \\
& T_{\eta}^{(2)}(0, x)=T_{\xi}^{(2)}\left(t_{*}, x\right),\left.T_{\eta}^{(2)}\right|_{x_{2}=0}=Q_{1}, \\
& \left.T_{\eta}^{(2)}\right|_{x_{2}=L_{2}}=Q_{2} ; \\
& z^{-1} \frac{\partial^{2} T_{\xi}^{(3)}}{\partial t^{2}}=\xi \frac{\partial}{\partial x_{3}}\left[A\left(T_{\eta}^{(2)}\right) \frac{\partial T_{\xi}^{(3)}}{\partial x_{3}}\right]+\xi \sigma_{3} A_{2}, \\
& 0<t<t_{*}, \\
& T_{\xi}^{(3)}(0, x)=T_{\eta}^{(2)}\left(t_{*}, x\right), \frac{\partial T_{\xi}^{(3)}(0, x)}{\partial t}=\frac{\partial T_{\eta}^{(2)}\left(t_{*}, x\right)}{\partial t},
\end{aligned}
$$




$$
\begin{gathered}
\left.T_{\xi}^{(3)}\right|_{x_{3}=0}=D_{1}\left(t_{*}, x_{1}, x_{2}\right), \\
\left.T_{\xi}^{(3)}\right|_{x_{3}=L_{3}}=D_{2}\left(t_{*}, x_{1}, x_{2}, L_{3}\right), \\
C\left(T_{\xi}^{(3)}\right) \frac{\partial T_{\eta}^{(3)}}{\partial t}=\eta \frac{\partial}{\partial x_{3}}\left[A\left(T_{\xi}^{(3)}\right) \frac{\partial T_{\eta}^{(3)}}{\partial x_{3}}\right]+ \\
Y_{3}\left(T_{\xi}^{(3)}\right) \frac{\partial T_{\eta}^{(3)}}{\partial x_{3}}+ \\
+\sigma_{3}\left[A_{1}\left(T_{\eta}^{(3)}\right)^{k}+\eta A_{2}\right], 0<t<t_{*}, \\
T_{\eta}^{(3)}(0, x)=T_{\xi}^{(3)}\left(t_{*}, x\right),\left.T_{\eta}^{(3)}\right|_{x_{3}=0}=D_{1}, \\
\left.T_{\eta}^{(3)}\right|_{x_{3}=L_{3}}=D_{2},
\end{gathered}
$$

where $\xi+\eta=1, \sigma_{1}+\sigma_{2}+\sigma_{3}=1$. When $C(T) \neq 0$ solved the system of equations (4) $-(15)$ for the telegraph equation: the presence of friction (conductive medium). When $C(T)=0, A_{1}=0, \eta=0$ solved the system of equations (4), (5), (8), (9), (12), (13) for the wave equation: the lack of friction (decaying environment). Indexes: $\mathrm{H}-$ the initial value, $*-$ characteristic value, 0 at the bottom - the ultimate value, $r$ - value of relaxation, $\xi$ - bottom part of the wave equation (1), $\eta$ - below the parabolic component of the equation (1).

We are talking about the next model, for example, the conductive-convective heat transfer for the hyperbolic heat equation at $A_{1}=A_{2}=0$. First, in the first stage is turned off by the conductive-convective heat transfer coordinate directions $x_{2}, x_{3}$, that is consider the problem (4) - (7). Then at $t=t_{*}$ we get a temperature distribution $T^{(1)}\left(t_{*}, x\right)$. Taking her by the initialing, turn off the conductive-convective heat transfer on directions $x_{1}, x_{3}$ and solve the problem (8) $-(11)$, then at $t=t_{*}$ we have the distribution $T^{(2)}\left(t_{*}, x\right)$. We take it for an initial temperature, turn off the conductive-convective heat transfer on directions $x_{1}, x_{2}$ and consider the problem (12) - (15). Then in moment $t=t_{*}$ we finally get the temperature $T^{(3)}\left(t_{*}, x\right)$, which coincides with the true value $T\left(t_{*}, x\right)$.

According to this model, the process the conductive-convective heat transfer "stretched" in time and takes place during the time of the gap $3 t_{*}$ [10], and instead $t_{*}$. Such an approach to solve multi-dimensional equations of partial differential equations with constant coefficients is proposed and justified in $[10,11]$. For the wave equation ( $\left.C(T)=0, A_{1}=0, \eta=0\right)$ are excluded by the coordinate directions $x_{1}, x_{2}, x_{3}$ - wave velocity, shear elasticity of the medium and so on.

But before that, to the system (4) - (15) must apply Kirchhoff transformation [4] and quasi-linearization [8,9] to obtain the differential equations with constant coefficients, which can be solved with Laplace integral transform [12].

In the future, to use the inversion formula $A(T)$ in (1) taken in the form

$$
A(T)=N T^{m}, m \geq 0, N>0, N=\mathrm{const}
$$

We use Kirchhoff transformation [4]

$$
v=\int_{0}^{T} \frac{A(T)}{A_{\mathrm{H}}} d T .
$$

Then, taking into account the relations [4]:

$$
\nabla A=\frac{\partial A}{\partial T} \nabla T, \frac{\partial v}{\partial t}=\frac{A}{A_{\mathrm{H}}} \frac{\partial T}{\partial t}, \nabla v=\frac{A}{A_{\mathrm{H}}} \nabla T,
$$

get out (4) - (18)

$$
\begin{aligned}
& v=T^{s} / \phi, \phi=s A_{\mathrm{H}} / N, s=m+1, \\
& b \frac{\partial}{\partial t}\left(\frac{A_{\mathrm{H}}}{A} \frac{\partial v_{\xi}^{(1)}}{\partial t}\right)=\frac{\partial^{2} v_{\xi}^{(1)}}{\partial x_{1}^{2}}+\sigma_{1} a_{2}, 0<t<t_{*}, \\
& \left.T_{\xi}^{(1)}\right|_{t=0}=p_{1}(x),\left.\frac{\partial T_{\xi}^{(1)}}{\partial t}\right|_{t=0}=p_{2}(x), 0<x_{j}<L_{j}, j \\
& =1,2,3 \text {, } \\
& \left.v_{\xi}^{(1)}\right|_{x_{1}=0}=g_{1},\left.v_{\xi}^{(1)}\right|_{x_{1}=L_{1}}=g_{2} \text {, } \\
& c_{1} \frac{\partial v_{\eta}^{(1)}}{\partial t}=\frac{\partial^{2} v_{\eta}^{(1)}}{\partial x_{1}^{2}}+r_{1} \frac{\partial v_{\eta}^{(1)}}{\partial x_{1}}+ \\
& +\sigma_{1}\left[a_{1}\left(T_{\eta}^{(1)}\right)^{k}+a_{2}\right], 0<t<t_{*}, \\
& v_{\eta}^{(1)}(0, x)=v_{\xi}^{(1)}\left(t_{*}, x\right),\left.v_{\eta}^{(1)}\right|_{x_{1}=0}=g_{1} \text {, } \\
& \left.v_{\eta}^{(1)}\right|_{x_{1}=L_{1}}=g_{2} ; \\
& b \frac{\partial}{\partial t}\left(\frac{A_{\mathrm{H}}}{A} \frac{\partial v_{\xi}^{(2)}}{\partial t}\right)=\frac{\partial^{2} v_{\xi}^{(2)}}{\partial x_{2}^{2}}+\sigma_{2} a_{2}, 0<t<t_{*}, \\
& T_{\xi}^{(2)}(0, x)=T_{\eta}^{(1)}\left(t_{*}, x\right), \frac{\partial T_{\xi}^{(2)}(0, x)}{\partial t}=\frac{\partial T_{\eta}^{(1)}\left(t_{*}, x\right)}{\partial t}, \\
& \left.v_{\xi}^{(2)}\right|_{x_{2}=0}=q_{1},\left.v_{\xi}^{(2)}\right|_{x_{2}=L_{2}}=q_{2}, \\
& c_{2} \frac{\partial v_{\eta}^{(2)}}{\partial t}=\frac{\partial^{2} v_{\eta}^{(2)}}{\partial x_{2}^{2}}+r_{2} \frac{\partial v_{\eta}^{(2)}}{\partial x_{2}}+
\end{aligned}
$$




$$
\begin{gathered}
+\sigma_{2}\left[a_{1}\left(T_{\eta}^{(2)}\right)^{k}+a_{2}\right], 0<t<t_{*}, \\
v_{\eta}^{(2)}(0, x)=v_{\xi}^{(2)}\left(t_{*}, x\right),\left.v_{\eta}^{(2)}\right|_{x_{2}=0}=q_{1}, \\
\left.v_{\eta}^{(2)}\right|_{x_{2}=L_{2}}=q_{2} ; \\
b \frac{\partial}{\partial t}\left(\frac{A_{\mathrm{H}}}{A} \frac{\partial v_{\xi}^{(3)}}{\partial t}\right)=\frac{\partial^{2} v_{\xi}^{(3)}}{\partial x_{3}^{2}}+\sigma_{3} a_{2}, 0<t<t_{*},(26) \\
T_{\xi}^{(3)}(0, x)=T_{\eta}^{(2)}\left(t_{*}, x\right), \frac{\partial T_{\xi}^{(3)}(0, x)}{\partial t}=\frac{\partial T_{\eta}^{(2)}\left(t_{*}, x\right)}{\partial t}, \\
\left.v_{\xi}^{(3)}\right|_{x_{3}=0}=d_{1},\left.\quad v_{\xi}^{(3)}\right|_{x_{3}=L_{3}}=d_{2}, \\
c_{3} \frac{\partial v_{\eta}^{(3)}}{\partial t}=\frac{\partial^{2} v_{\xi}^{(3)}}{\partial x_{3}^{2}}+r_{3} \frac{\partial v_{\eta}^{(3)}}{\partial x_{3}}+ \\
+\sigma_{3}\left[a_{1}\left(T_{\eta}^{(3)}\right)^{k}+a_{2}\right], 0<t<t_{*}, \\
v_{\eta}^{(3)}(0, x)=v_{\xi}^{(3)}\left(t_{*}, x\right),\left.v_{\eta}^{(3)}\right|_{x_{3}=0}=d_{1} \\
\left.v_{\eta}^{(3)}\right|_{x_{3}=L_{3}}=d_{2},
\end{gathered}
$$

where $c_{i}=C\left(T_{\xi}^{(i)}\right) /(\eta A), \quad r_{i}=Y_{i} /(\eta A), i=1,2,3$, $b=1 /\left(\xi c^{2}\right), a_{1}=A_{1} /\left(\eta A_{\mathrm{H}}\right), a_{2}=A_{2} / A_{\mathrm{H}}, g_{i}=G_{i}^{s} / \phi$, $q_{i}=Q_{i}^{s} / \phi, d_{i}=D_{i}^{s} / \phi, i=1,2$.

This intermediate value in directions: $T_{\xi}^{(j)}\left(t_{*}, x\right)$, $T_{\eta}^{(j)}\left(t_{*}, x\right), j=1,2,3$ determined from (19) the inversion formulas

$$
T_{\xi}^{(j)}=\left(\phi v_{\xi}^{(j)}\right)^{1 / s}, T_{\eta}^{(j)}=\left(\phi v_{\eta}^{(j)}\right)^{1 / s}, j=1,2,3 .
$$

Then the final solution of the problem (1) - (3) is write

$$
T\left(t_{*}, x\right)=T_{\eta}^{(3)}\left(t_{*}, x\right) .
$$

Note that the range of the independent variables and the type of boundary conditions do not change under the transformation Kirchhoff (17), and in the presence of the inversion formula boundary conditions of the first kind of goes to the Dirichlet condition.

Our purpose to receive a solution of the nonlinear boundary value problem, if it exists, as the limit of a sequence of solutions of linear boundary value problems. To do this, we use the results [7-9]. Assume further that all the coordinate directions in space are equivalent. Let $v^{(1)}=$ const there is an initial approximation [as an initial approximation to take for $v^{(1)}$ the first formula of (21) with the first equation in (19)]. For simplicity, consider the case of a quasi-one dimension analysis and sequence $v_{n}(t, x)$ defined by the recurrence relation [8] (dot and bar at the top indicate the partial derivative with respect to time and space)

$$
\begin{gathered}
\frac{\partial^{2} v_{n+1}}{\partial y^{2}}=f+\left(v_{n+1}-v_{n}\right) \frac{\partial f}{\partial v_{n}}+\left(v_{n+1}^{\prime}-v_{n}^{\prime}\right) \frac{\partial f}{\partial v_{n}^{\prime}}+ \\
\left(\dot{v}_{n+1}-\dot{v}_{n}\right) \frac{\partial f}{\partial \dot{v}_{n}}, \\
f=f\left(v_{n}, v_{n}^{\prime}, \dot{v}_{n}\right), v_{\text {н }}=v_{n+1}(0, x),\left.v_{n+1}\right|_{\Gamma}=\Psi^{s} / \phi, \\
n=0,1,2, \ldots,
\end{gathered}
$$

where $y$ there is the any coordinate from $x_{j}, j=1,2,3$ in (29). Then for $y=x_{1}$ the rest coordinates in (29) $0<x_{j}<L_{j}, j=2,3$ change parametrically. The remaining coordinates the preparation of expressions (29) and (30) occurs circular replacement index if instead substitute $\mathrm{y}$ respectively $x_{2}, x_{3}$. Note that in solving the three-dimensional boundary value problem (20) - (26), while in the first coordinate $x_{1}$ in the direction of an initial iteration acts $v_{n}$, the subsequent iteration $v_{n+1}$ is obtained from the final expression $v_{n+1}\left(t_{*}, x\right)=v^{(3)}\left(t_{*}, x\right)$ [see comment below to formulas (56) - (61)]. Then in a quasi-one dimension of equation (29), (30) can be rewritten in the coordinate $x_{1}[7]$ :

$$
\begin{gathered}
\frac{\partial^{2} v^{(1)}}{\partial x_{1}^{2}}=f_{1}+\left(v^{(1)}-v^{(0)}\right) \frac{\partial f_{1}}{\partial v^{(0)}}+\left(v^{\prime(1)}-v^{(0)}\right) \frac{\partial f_{1}}{\partial v^{(0)}} \\
+\left(\dot{v}^{(1)}-\dot{v}^{(0)}\right) \frac{\partial f_{1}}{\partial \dot{v}^{(0)}} \\
f_{1}=f_{1}\left(v^{(0)}, v^{(0)}, \dot{v}^{(0)}\right), v^{(1)}(0, x)=v^{(0)}\left(t_{*}, x\right), \\
v^{(0)}=v_{\mathrm{H}}, \\
\left.v^{(1)}\right|_{x_{1}=0}=g_{1},\left.\quad v^{(1)}\right|_{x_{1}=L_{1}}=g_{2} .(32)
\end{gathered}
$$

Expressions similar to (29), (30) can be written in other coordinate directions $x_{2}, x_{3}$. In particular, the second coordinate direction $x_{2}$ is necessary in (31), (32) everywhere to replace the top and bottom indexes (1) and 1 on (2) and 2, and the top index (0) on (1). Thus for the entry condition in the second co-ordinate direction $x_{2}$ have $v^{(2)}(0, x)=v^{(1)}\left(t_{*}, x\right)$.

Each function $v_{n+1}$ in (29), (30) in the case of quasi-one dimension or $v^{(1)}$ in (31), (32) is solution of the linear equation, which is a very important feature of this algorithm. The algorithm follows from the approximation method of Newton-Kantorovich [9] in the functional space.

In order to reduce further records introduce the following notation: 


$$
\begin{gathered}
R_{\xi}^{(i)}=\sigma_{i} a_{2}, \\
f_{i}=c_{i} \dot{v}_{\eta}^{(i-1)}-r_{i} \partial v_{\eta}^{(i-1)} / \partial x_{i}-\sigma_{i}\left(a_{1} Z_{i}^{k / s}+a_{2}\right) \\
Z_{i}=\left(\phi v_{\eta}^{(i-1)}\right), c_{i}\left(v_{\eta}^{(i-1)}\right)=\partial f_{i} / \partial \dot{v}_{\eta}^{(i-1)}, \\
r_{i}=-\partial f_{i} / \partial v_{\eta}^{(i-1)}, \\
\Phi_{i}=\sigma_{i} a_{1} k Z_{i}^{k / s} A_{\mathrm{H}} /\left(Z_{i} N\right), \Phi_{i}=-\partial f_{i} / \partial v_{\eta}^{(i-1)} \\
R_{\eta}^{(i)}=\sigma_{i} a_{1} Z_{i}^{k / s}\left[1-v_{\eta}^{(i-1)} k A_{\mathrm{H}} /\left(Z_{i} N\right)\right]+\sigma_{i} a_{2}, \\
h_{\eta}^{(i)}=c_{i} \dot{v}_{\eta}^{(i)}-R_{\eta}^{(i)}, i=1,2,3 .
\end{gathered}
$$

Obtain a quasi-one dimension solution of the problem (31), (32) in coordinate direction $x_{1}$, using equation (20) - (22)

$$
\begin{gathered}
\frac{\partial^{2} v_{\xi}^{(1)}}{\partial x_{1}^{2}}=h_{1}, h_{1}=b \frac{\partial}{\partial t}\left(\frac{A_{\mathrm{H}}}{A} \frac{\partial v_{\xi}^{(1)}}{\partial t}\right)-R_{\xi}^{(1)}, 0<t<t_{*}, \\
\left.T_{\xi}^{(1)}\right|_{t=0}=p_{1}(x),\left.\frac{\partial T_{\xi}^{(1)}}{\partial t}\right|_{t=0}=p_{2}(x), \\
\left.v_{\xi}^{(1)}\right|_{x_{1}=0}=g_{1},\left.v_{\xi}^{(1)}\right|_{x_{1}=L_{1}}=g_{2} .
\end{gathered}
$$

Laplace integral transform is applicable to the differential equation (34), except for derivative on $x_{1}$ and replacing it with a linear expression relative to the image of the desired function. In what follows we consider functions for which the Laplace integral transform is absolutely convergent. The real part of the complex number $p=\alpha+i \beta, i=\sqrt{-1}$ is positive, that is $\operatorname{Re} p>0$. We denote the image in big letters $V^{(1)}, H_{1}$. It is assumed that in calculating the image coordinates $x_{i}, i=1,2,3$, we operate with the functions of the analytic continuation to the values $x_{i}>L_{i}$ on that law, which they are defined in the interval $\left(0, L_{i}\right), i=1,2,3$.

It is believed that the solution $v^{(1)}(t, x)$ and its derivatives in equation (34), satisfy the conditions for the existence of Laplace integral transform on $x_{1}$, and the degree of growth on $x_{1}$ the function $v^{(1)}$ and its derivatives do not depend from $t, x_{2}, x_{3}$. For simplicity, we omit the index calculations $*$ at $t$ at the bottom of the index $\xi$ at $v_{\xi}^{(1)}$, as well as the index of (1) at the top and at the bottom at $\quad v_{\xi}^{(1)}, V^{(1)}, h_{1}, H_{1} \quad$, indicating $\partial v_{\xi}^{(1)}\left(t, 0, x_{2}, x_{3}\right) / \partial x_{1}=\partial g_{1} / \partial x_{1}$, then we have from (34) [12]:

$$
p^{2} V\left(t, p, x_{2}, x_{3}\right)-p g_{1}-\frac{\partial g_{1}}{\partial x_{1}}=H\left(t, p, x_{2}, x_{3}\right),
$$

$$
\begin{gathered}
0<x_{j}<L_{j}, j=2,3 \\
V\left(t, p, x_{2}, x_{3}\right)=\frac{g_{1}}{p}+\frac{\partial g_{1} / \partial x_{1}}{p^{2}}+\frac{H}{p^{2}} .
\end{gathered}
$$

Using the inverse Laplace integral transform [12]: $\mathrm{L}^{-1}\left(1 / p^{2}\right)=x_{1}, \mathrm{~L}^{-1}[H(p) / p]=\int_{0}^{x_{1}} h(y) d y$, to restore the original для $v(t, x)$ from (37) [12]

$$
v(t, x)=g_{1}+x_{1} \frac{\partial g_{1}}{\partial x_{1}}+\int_{0}^{x_{1}}\left(x_{1}-y\right) h(y) d y
$$

The derivative $\partial g_{1} / \partial x_{1}$ in (38) we find, using the second boundary condition of (36)

$$
g_{2}=g_{1}+L_{1} \frac{\partial g_{1}}{\partial x_{1}}+\int_{0}^{L_{1}}\left(L_{1}-y\right) h(y) d y
$$

Therefore, finding $\partial g_{1} / \partial x_{1}$ in (39) and substituting it into (38), we obtain

$$
\begin{aligned}
v(t, x)=g_{1}+\frac{x_{1}}{L_{1}} & {\left[g_{2}-g_{1}-\int_{0}^{L_{1}}\left(L_{1}-y\right) h(y) d y\right] } \\
& +\int_{0}^{x_{1}}\left(x_{1}-y\right) h(y) d y
\end{aligned}
$$

Transform the expression on the right-hand side of (40) so as to get rid of the integral with variable upper limit. Then, by introducing the Green's function $E_{1}\left(x_{1}, y\right)$ [7,8]

$$
E_{1}\left(x_{1}, y\right)=\left\{\begin{array}{l}
y\left(L_{1}-x_{1}\right) / L_{1}, 0 \leq y \leq x_{1} \\
x_{1}\left(L_{1}-y\right) / L_{1}, x_{1} \leq y \leq L_{1}
\end{array}\right.
$$

expression (40) is rewritten in the return of the upper index (1) and lower $\xi$, noting that $b, A$ is clearly not dependent on $x_{1}$, unlike $R_{\xi}^{(1)}$ in (33)

$$
\begin{gathered}
b \frac{\partial}{\partial t}\left(\frac{A_{\mathrm{H}}}{A} \frac{\partial v_{\xi}^{(1)}}{\partial t}\right) \int_{0}^{L_{1}} E_{1}\left(x_{1}, y\right) d y+v_{\xi}^{(1)}= \\
=\left[g_{1}+\frac{x_{1}}{L_{1}}\left(g_{2}-g_{1}\right)+\int_{0}^{L_{1}} E_{1}\left(x_{1}, y\right) R_{\xi}^{(1)} d y\right]=F_{1} .
\end{gathered}
$$

We apply again Kirchhoff transformation (17) to return to the original variable $T$ in equation (4)

$$
T=\int_{0}^{v} \frac{A_{\mathrm{H}}}{A} d v, A=N T^{m}, T=(\phi v)^{1 / s} .
$$


Transform the left side of equation (42), using (43)

$$
\begin{gathered}
\frac{\partial T}{\partial t}=\frac{A_{\mathrm{H}}}{A} \frac{\partial v}{\partial t}, \frac{\partial^{2} T}{\partial t^{2}}=\frac{\partial}{\partial t}\left(\frac{A_{\mathrm{H}}}{A} \frac{\partial v}{\partial t}\right), v=T^{s} / \phi, \\
\phi=s A_{\mathrm{H}} / N, s=m+1 .
\end{gathered}
$$

Since the second term on the left-hand side of equation (42) takes the form $v_{\xi}^{(1)}=\left(T_{\xi}^{(1)}\right)^{s} / \phi$, then it is necessary to reapply the quasi-linearization (31), (32), then we have

$$
\begin{gathered}
\frac{\partial^{2} T_{\xi}^{(1)}}{\partial t^{2}}+\frac{s}{\phi} B_{1}\left(T_{\xi}^{(0)}\right)^{m} T_{\xi}^{(1)}= \\
=\left[Y_{1}^{-1} F_{1}+(s / \phi-1) B_{1}\left(T_{\xi}^{(0)}\right)^{s}\right]=P_{1}, \\
Y_{1}=b \int_{0}^{L_{1}} E_{1}\left(x_{1}, y\right) d y,
\end{gathered}
$$

где, $B_{1}=Y_{1}^{-1}, B_{1}>0, T_{\xi}^{(0)}=p_{1}(x), E_{1}\left(x_{1}, y\right)>0$ из

We use the idea of Doetsch [13] on the applicability of Laplace integral transform to the partial differential equation as many times as its dimension. Then, with the initial conditions (35), we obtain $[7,12]$ of $(44)$

$$
\begin{gathered}
T_{\xi}^{(1)}\left(t_{*}, x\right)=s_{1}\left(t_{*}\right) p_{1}(x)+s_{2}\left(t_{*}\right) p_{2}(x)+ \\
\int_{0}^{t_{*}} s_{2}\left(t_{*}-\tau\right) P_{1}(\tau, x) d \tau \\
s_{2}\left(t_{*}\right)=\gamma_{1}^{-1} \sin \left(\gamma_{1} t_{*}\right), \\
s_{2}\left(t_{*}-\tau\right)=\gamma_{1}^{-1} \sin \left[\gamma_{1}\left(t_{*}-\tau\right)\right] \\
s_{1}\left(t_{*}\right)=\cos \left(\gamma_{1} t_{*}\right), s_{1}\left(t_{*}-\tau\right)=\cos \left[\gamma_{1}\left(t_{*}-\tau\right)\right], \\
\frac{\partial T_{\xi}^{(1)}\left(t_{*}, x\right)}{\partial t}=\sqrt{\beta_{1}}, \beta_{1}=s B_{1}\left(T_{\xi}^{(0)}\right)^{m} / \phi \\
\gamma_{1}\left(t_{*}\right) p_{2}(x)-\gamma_{1}^{2} s_{2}\left(t_{*}\right) p_{1}(x)+ \\
+\int_{0}^{t_{*}} s_{1}\left(t_{*}-\tau\right) P_{1}(\tau, x) d \tau .
\end{gathered}
$$

Now we obtain an analytical solution of the problem of the intermediate (23), (24), using the notation (33)

$$
\frac{\partial^{2} v_{\eta}^{(1)}}{\partial x_{1}^{2}}+\Phi_{1} v_{\eta}^{(1)}=h_{\eta}^{(1)}-r_{1} \frac{\partial v_{\eta}^{(1)}}{\partial x_{1}}, 0<t<t_{*},
$$

$v_{\eta}^{(1)}(0, x)=v_{\xi}^{(1)}\left(t_{*}, x\right),\left.v_{\eta}^{(1)}\right|_{x_{1}=0}=g_{1},\left.v_{\eta}^{(1)}\right|_{x_{1}=L_{1}}=g_{2}$.

Note that $\Phi_{1}$ of (33) does not depend explicitly of $x_{1}$ in (48) $\left[\Phi_{1}\right.$ you can always ask at the bottom of iteration $\mathrm{n}$, knowing the value $T_{\xi}^{(1)}$ of the initial and subsequent times from (45) and $v_{\xi}^{(1)}$ from (19)]. For simplicity, we omit the index calculations $*$ at $\mathrm{t}$ the bottom of the index $\eta$ at $v_{\eta}^{(1)}$, and as well as the index of (1) at the top and at the bottom at $v_{\eta}^{(1)}, V^{(1)}, h_{\eta}^{(1)}, H_{1}$. Then, as in (34) - (41) we have

$$
\begin{gathered}
p^{2} V\left(t, p, x_{2}, x_{3}\right)-p g_{1}-\frac{\partial g_{1}}{\partial x_{1}}+\Phi_{1} V\left(t, p, x_{2}, x_{3}\right) \\
+r_{1}\left[p V\left(t, p, x_{2}, x_{3}\right)-g_{1}\right]=H\left(t, p, x_{2}, x_{3}\right) \\
0<x_{j}<L_{j}, j=2,3, \\
V=\frac{(p+\delta) g_{1}}{(p+\delta)^{2}+b_{1}^{2}}+\frac{b_{1}\left(\delta g_{1}+\partial g_{1} / \partial x_{1}+H\right)}{b_{1}\left[(p+\delta)^{2}+b_{1}^{2}\right]},(49)
\end{gathered}
$$

where $\delta=r_{1} / 2, b_{1}=\sqrt{\Phi_{1}-\delta^{2}}$.

Using the inverse Laplace integral transform [12]: $\mathrm{L}^{-1}\left[p /\left(p^{2}+b_{1}^{2}\right)\right]=\cos \left(b_{1} x_{1}\right)$ at $b_{1}^{2}=\Phi_{1}-\delta^{2}>0$, $\mathrm{L}^{-1}\left[p /\left(p^{2}-b_{1}^{2}\right)\right]=\cosh \left(b_{1} x_{1}\right) \quad$ at $b_{1}^{2}<0$, $\mathrm{L}^{-1}\left[(p+\delta)^{-1}\right]=\exp \left(-\delta x_{1}\right)$

$\mathrm{L}^{-1}[H(p) / p]=\int_{0}^{x_{1}} h(y) d y$, to restore the original for $v(t, x)$ of (49) [12]

$$
\begin{gathered}
v(t, x)=\exp \left(-\delta x_{1}\right) \\
\left\{g_{1}\left[u_{1}\left(x_{1}\right)+\delta u_{2}\left(x_{1}\right)\right]+u_{2}\left(x_{1}\right) \partial g_{1} / \partial x_{1}\right\}+(50) \\
+\exp \left(-\delta x_{1}\right) \int_{0}^{x_{1}} \exp (\delta y) u_{2}\left(x_{1}-y\right) h(y) d y, \\
0<x_{j}<L_{j}, j=2,3 ; \\
u_{2}\left(x_{1}\right)=b_{1}^{-1} \sin \left(b_{1} x_{1}\right), \\
u_{2}\left(x_{1}-y\right)=b_{1}^{-1} \sin \left[\left(b_{1}\left(x_{1}-y\right)\right],(51)\right. \\
u_{1}\left(x_{1}\right)=\cos \left(b_{1} x_{1}\right), b_{1}^{2}=\Phi_{1}-\delta^{2}>0 ; \\
u_{1}\left(x_{1}\right)=\cosh \left(b_{1} x_{1}\right), u_{2}\left(x_{1}\right)=b_{1}^{-1} \sinh \left(b_{1} x_{1}\right), \\
u_{2}\left(x_{1}-y\right)=b_{1}^{-1} \sinh \left[\left(b_{1}\left(x_{1}-y\right)\right], b_{1}^{2}<0 .\right.
\end{gathered}
$$

We find the derivative $\partial g_{1} / \partial x_{1}$ in (50) as in (39), using the second boundary condition in (48) and substitute it into equation (50), we obtain 


$$
\begin{gathered}
v(t, x)=\exp \left(-\delta x_{1}\right) g_{1}\left[u_{1}\left(x_{1}\right)+\delta u_{2}\left(x_{1}\right)\right]+(52) \\
+\exp \left(-\delta x_{1}\right) \int_{0}^{x_{1}} \exp (\delta y) u_{2}\left(x_{1}-y\right) h(y) d y-\frac{u_{2}\left(x_{1}\right)}{u_{2}\left(L_{1}\right)} \times \\
\times \exp \left(-\delta x_{1}\right) \int_{0}^{L_{1}} \exp (\delta y) u_{2}\left(L_{1}-y\right) h(y) d y+ \\
\times u_{2}\left(x_{1}\right)\left\{g_{2} \exp \left(\delta L_{1}\right)-\left[u_{1}\left(L_{1}\right)+\delta u_{2}\left(L_{1}\right)\right] g_{1}\right\} / \\
u_{2}\left(L_{1}\right) .
\end{gathered}
$$

Transform the expression on the right-hand side of (52) so as to get rid of the integral with variable upper limit. Then, by introducing the Green's function $G_{1}\left(x_{1}, y\right)[7,8]$

$$
\begin{gathered}
G_{1}\left(x_{1}, y\right)= \\
\left\{\begin{array}{l}
\exp \left[\delta\left(y-x_{1}\right)\right]\left[u_{2}\left(x_{1}\right) u_{2}\left(L_{1}-y\right) / u_{2}\left(L_{1}\right)-\right. \\
\left.-u_{2}\left(x_{1}-y\right)\right], 0 \leq y \leq x_{1}, \\
\exp \left[\delta\left(y-x_{1}\right)\right] u_{2}\left(x_{1}\right) u_{2}\left(L_{1}-y\right) / u_{2}\left(L_{1}\right), \\
x_{1} \leq y \leq L_{1},
\end{array}\right.
\end{gathered}
$$

expression (51), using the formulas (33) and the return of a subscript $*, \eta$, as well as the top - (1), is rewritten

$$
\begin{gathered}
\dot{v}_{\eta}^{(1)}+U_{1} v_{\eta}^{(1)}=X_{1}^{-1}\left[S_{1}\left(t_{*}, x\right)+\int_{0}^{L_{1}} G_{1}\left(x_{1}, y\right) R_{\eta}^{(1)} d y\right]= \\
W_{1}\left(t_{*}, x\right), \\
S_{1}\left(t_{*}, x\right)=\exp \left(-\delta x_{1}\right) g_{1}\left[u_{1}\left(x_{1}\right)+\delta u_{2}\left(x_{1}\right)\right]+u_{2}\left(x_{1}\right) \\
\quad \exp \left(-\delta x_{1}\right) \times \\
\times\left\{g_{2} \exp \left(\delta L_{1}\right)-\left[u_{1}\left(L_{1}\right)+\delta u_{2}\left(L_{1}\right)\right] g_{1}\right\} / u_{2}\left(L_{1}\right), \\
X_{1}=\int_{0}^{L_{1}} G_{1}\left(x_{1}, y\right) c_{1}\left(v_{\xi}^{(1)}\right) d y, U_{1}=X_{1}^{-1}, \\
v_{\eta}^{(1)}(0, x)=v_{\xi}^{(1)}\left(t_{*}, x\right) .
\end{gathered}
$$

As a result, the solution of (54) takes the form [14]:

$$
\begin{gathered}
v_{\eta}^{(1)}\left(t_{*}, x\right)=\left[v_{\xi}^{(1)}\left(t_{*}, x\right)+\int_{0}^{t_{*}} W_{1}(x, \tau) \exp \left(\tau U_{1}\right) d \tau\right] \times(55) \\
\times \exp \left(-t_{*} U_{1}\right), 0<x_{j}<L_{j}, j=2,3
\end{gathered}
$$

where $v_{\xi}^{(1)}\left(t_{*}, x\right)$ is taken from the formula (45) using equation (19).
Similarly, solutions are obtained quasi one-dimensional problems (25), (26) on the coordinate directions $x_{2}, x_{3}$ with the involvement of (31) - (33) and the inversion formula (27). Then, according to the algorithm (37) - (55) at $\partial^{2} T_{\eta}^{(i)}(t, x) / \partial t^{2}=0, i=1,2,3$ в (6), (10), (14) (these derivatives were not present) we have

$$
\begin{aligned}
& T_{\xi}^{(i)}\left(t_{*}, x\right)= \\
& s_{1}\left(t_{*}\right) T_{\eta}^{(i-1)}\left(t_{*}, x\right)+s_{2}\left(t_{*}\right) \frac{\partial T_{\eta}^{(i-1)}\left(t_{*}, x\right)}{\partial t}+ \\
& +\int_{0}^{t_{*}} s_{2}\left(t_{*}-\tau\right) P_{i}(\tau, x) d \tau, \\
& P_{i}=Y_{i}^{-1} F_{i}+B_{i}(s / \phi-1)\left(T_{\eta}^{(i-1)}\right)^{s}, i=2,3, \\
& T_{\xi}^{(i)}\left(t_{*}, x\right)= \\
& s_{1}\left(t_{*}\right) T_{\xi}^{(i-1)}\left(t_{*}, x\right)+s_{2}\left(t_{*}\right) \frac{\partial T_{\xi}^{(i-1)}\left(t_{*}, x\right)}{\partial t}+(57) \\
& +\int_{0}^{t_{*}} s_{2}\left(t_{*}-\tau\right) P_{i}(\tau, x) d \tau, \\
& P_{i}=Y_{i}^{-1} F_{i}+B_{i}(s / \phi-1)\left(T_{\xi}^{(i-1)}\right)^{s}, \\
& Y_{i}=b \int_{0}^{L_{i}} E_{i}\left(x_{i}, y\right) d y, B_{i}=Y_{i}^{-1}, i=2,3, \\
& F_{2}=\left[q_{1}+\frac{x_{2}}{L_{2}}\left(q_{2}-q_{1}\right)+\int_{0}^{L_{2}} E_{2}\left(x_{2}, y\right) R_{\xi}^{(2)} d y\right] \text {, } \\
& F_{3}=\left[d_{1}+\frac{x_{3}}{L_{3}}\left(d_{2}-d_{1}\right)+\int_{0}^{L_{3}} E_{3}\left(x_{3}, y\right) R_{\xi}^{(3)} d y\right] \text {, } \\
& \frac{\partial T_{\xi}^{(i)}\left(t_{*}, x\right)}{\partial t}=2 \\
& s_{1}\left(t_{*}\right) \frac{\partial T_{\eta}^{(i-1)}\left(t_{*}, x\right)}{\partial t}-\gamma_{i}^{2} s_{2}\left(t_{*}\right) T_{\eta}^{(i-1)}\left(t_{*}, x\right)
\end{aligned}
$$$$
+\int_{0}^{t_{*}} s_{1}\left(t_{*}-\tau\right) P_{i}(\tau, x) d \tau,
$$$$
P_{i}=Y_{i}^{-1} F_{i}+B_{i}(s / \phi-1)\left(T_{\eta}^{(i-1)}\right)^{s}, i=2,3,
$$$$
\frac{\partial T_{\xi}^{(i)}\left(t_{*}, x\right)}{\partial t}=2
$$ 


$$
\begin{aligned}
& s_{1}\left(t_{*}\right) \frac{\partial T_{\xi}^{(i-1)}\left(t_{*}, x\right)}{\partial t}-\gamma_{i}^{2} s_{2}\left(t_{*}\right) T_{\xi}^{(i-1)}\left(t_{*}, x\right) \\
& +s_{2}\left(t_{*}\right) B_{i-1}\left[F_{i-1}-\left(T_{\xi}^{(i-1)}\right)^{s} / \phi\right]+ \\
& \int_{0}^{t_{*}} s_{1}\left(t_{*}-\tau\right) P_{i}(\tau, x) d \tau, \\
& P_{i}=Y_{i}^{-1} F_{i}+B_{i}(s / \phi-1)\left(T_{\xi}^{(i-1)}\right)^{s}, i=2,3, \\
& \frac{\partial T_{\eta}^{(i-1)}\left(t_{*}, x\right)}{\partial t}=\frac{A_{\mathrm{H}}\left(T_{\eta}^{(i-1)}\right)^{-m}}{N} \frac{\partial v_{\eta}^{(i-1)}\left(t_{*}, x\right)}{\partial t},(60) \\
& \frac{\partial v_{\eta}^{(i-1)}\left(t_{*}, x\right)}{\partial t}=W_{i-1}\left(t_{*}, x\right)-U_{i-1} v_{\eta}^{(i-1)}\left(t_{*}, x\right), i=2,3, \\
& v_{\eta}^{(i)}\left(t_{*}, x\right)=\left[v_{\xi}^{(i)}\left(t_{*}, x\right)+\int_{0}^{t_{*}} W_{i} \exp \left(\tau U_{i}\right) d \tau\right] \times \\
& \times \exp \left(-t_{*} U_{i}\right), i=2,3, \\
& W_{i}=X_{i}^{-1}\left[S_{i}\left(t_{*}, x\right)+\int_{0}^{L_{i}} G_{i}\left(x_{i}, y\right) R_{\eta}^{(i)} d y\right], U_{i}=X_{i}^{-1}, \\
& X_{i}=\int_{0}^{L_{i}} G_{i}\left(x_{i}, y\right) c_{i}\left(v_{\xi}^{(i)}\right) d y, v_{\eta}^{(i)}(0, x)=v_{\xi}^{(i)}\left(t_{*}, x\right), i= \\
& \text { 2, } 3 \text {, } \\
& S_{2}\left(t_{*}, x\right)=\exp \left(-\delta x_{2}\right) q_{1}\left[u_{1}\left(x_{2}\right)+\delta u_{2}\left(x_{2}\right)\right]+ \\
& u_{2}\left(x_{2}\right) \exp \left(-\delta x_{2}\right) \times \\
& \times\left\{q_{2} \exp \left(\delta L_{2}\right)-\left[u_{1}\left(L_{2}\right)+\delta u_{2}\left(L_{2}\right)\right] q_{1}\right\} / u_{2}\left(L_{2}\right), \\
& S_{3}\left(t_{*}, x\right)=\exp \left(-\delta x_{3}\right) d_{1}\left[u_{1}\left(x_{3}\right)+\delta u_{2}\left(x_{3}\right)\right]+ \\
& u_{2}\left(x_{3}\right) \exp \left(-\delta x_{3}\right) \times \\
& \times\left\{d_{2} \exp \left(\delta L_{3}\right)-\left[u_{1}\left(L_{3}\right)+\delta u_{2}\left(L_{3}\right)\right] d_{1}\right\} / u_{2}\left(L_{3}\right),
\end{aligned}
$$
$T_{n+1}\left(t_{*}, x\right)=T_{\eta}^{(3)}\left(t_{*}, x\right)$, then the iterative process is included $n=0,1,2 \ldots$.

Using the results of $[7,15]$, we find the condition for the unique solvability of the problem (1) - (3) under certain assumptions, and get a quadratic rate of convergence of the iterative process.

\section{Test Results of Inspections}

The accuracy of the analytical formulas (19), (27), (45) (47), (55) - (61) check on the test function in the solution of boundary value problems for partial differential equations in $\bar{Q}_{t}:\left[0 \leq x_{j} \leq L_{j}, j=1,2,3\right] \times\left[0<t \leq t_{0}\right]$ at $A(T)$ $=N T^{m}, m>0$

$$
\begin{aligned}
z^{-1} \frac{\partial^{2} T}{\partial t^{2}}+A_{3} \frac{\partial T}{\partial t} & =\sum_{j=1}^{3}\left\{\frac{\partial}{\partial x_{j}}\left[A(T) \frac{\partial T}{\partial x_{j}}\right]+C_{2} \frac{\partial T}{\partial x_{j}}\right\}+(62) \\
& +A_{1} T^{k}+F(x, t)
\end{aligned}
$$

with initial conditions

$$
\left.T\right|_{t=0}=\exp (y),\left.\frac{\partial T}{\partial t}\right|_{t=0}=w^{-1} \exp (y), w=\text { const, } w>
$$

$$
y=\sum_{i=1}^{3} z_{i}, z_{i}=\frac{x_{i}}{L_{i}}, i=1,2,3
$$

where the formulas (56), (58), (60), (61) are used to solve the telegraph equation, and the formulas (57), (59) are used to solve wave equation $\left(C(T)=A_{1}=0, \eta=0\right)$. Thus $s_{1}\left(t_{*}\right)$, $s_{2}\left(t_{*}\right) s_{2}\left(t_{*}-\tau\right), s_{1}\left(t_{*}-\tau\right)$ are taken from (46) with the change in the index 1 at $\gamma_{1}, \beta_{1}, B_{1}$ in order of sequence anywhere on the index 2 and 3 .

Similarly obtained $E_{i}\left(x_{i}, y\right), G_{i}\left(x_{i}, y\right)$ from (41), (53) and $u_{1}\left(x_{i}\right), u_{2}\left(x_{i}\right), u_{2}\left(x_{i}-y\right), i=2,3$ from (51) by replacing all arguments $x_{1}, L_{1}$ respectively in order to follow $x_{i}, L_{i}, i=2,3$. At $x=x_{2}$ in (56) - (61) other variables $0<x_{i}<L_{i}, i=1,3$ parametrically modified as in
(55). A similar situation is for $x=x_{3}$, this gives a final conditions (2), (3) the inversion formula (27): $T_{n+1}\left(t_{*}, x\right)=$ $T_{\eta}^{(3)}\left(t_{*}, x\right), \forall t_{*}>0, n=0,1,2 \ldots$

As can be seen from the algorithm, first intermediate values are excluded $T_{\xi}^{(i)}, v_{\xi}^{(i)}, T_{\eta}^{(i)}, v_{\eta}^{(i)}, i=1,2,3$ of the equations (19) and (27), (45) - (47), (55) - (61) and formed of the formula $(56)-(61)$ to $v_{n+1}\left(t_{*}, x\right)=v_{\eta}^{(3)}\left(t_{*}, x\right)$, then treatment of the formula (27) - final expression (28): 
It was taken the exact solution (62) - (64)

$$
T=\exp (\tau+y)
$$

then the source of $F$ in (62) has the form

$$
\begin{gathered}
F=\exp (\tau+y) \\
\left\{z^{-1} / w^{2}+A_{3} / w-C_{2}\left(L_{1}^{-1}+L_{2}^{-1}+L_{3}^{-1}\right)-\right. \\
\left.-s\left(L_{1}^{-2}+L_{2}^{-2}+L_{3}^{-2}\right) N \exp [m(\tau+y)]\right\}-A_{1} \\
\exp [k(\tau+y)] .
\end{gathered}
$$

The following reference values were used input: $m=0.5$, $w=1, \xi=\eta=0.5, \sigma_{1}=0.4, \sigma_{2}=0.3, \sigma_{3}=0.3$, $t_{0}=10^{-3} \mathrm{~s}, L_{i}=0.1 \mathrm{~m}, i=1,2,3, A_{\mathrm{H}}=N=0.0253$ $\mathrm{W} /(\mathrm{m} \cdot \mathrm{K}), c=340 \mathrm{~m} / \mathrm{s}, \quad A_{3}=1.3 \times 10^{3} \mathrm{~J} /\left(\mathrm{m}^{3} \cdot \mathrm{K}\right)$ (air environment [16]); $N_{i}=11, \Delta x_{i}=L_{i} /\left(N_{i}-1\right), i=1$, 2, 3, $\Delta t=t_{0} /(M-1), M=11$ - estimated number of units and the steps in space and time in finding the integrals in equations (42), (45) - (47), (55) - (61) by Simpson [17].

The boundary value problem (62) - (64) was solved with the help of formulas (19), (27), (45) - (47), (55) - (61). The number of iterations was monitored for the final expression in $(56)-(61)$ for $i=3$ to the relative change in the vector of error:

$$
\left\|V_{n}\right\|=\max _{x, t \in \bar{Q}_{t}}\left|\frac{v_{n+1}-v_{n}}{v_{n+1}}\right|
$$

Numerical examples are given for $\left\|V_{n}\right\| \leq \delta, \delta=0.01$. At the same time took only two iterations to achieve this accuracy and computation time of any version of $t_{c}=2 \mathrm{~s}$.

\begin{tabular}{|c|c|c|c|c|}
\hline № & $A_{1}$ & $C_{2}$ & $k$ & $\varepsilon, \%$ \\
\hline 1 & 1 & -6.5 & 1 & 0.085 \\
\hline 2 & 1 & 13 & 1 & 0.242 \\
\hline 3 & 1 & -13 & 1 & 0.083 \\
\hline 4 & 1 & 13 & 2 & 0.297 \\
\hline 5 & 2 & 13 & 2 & 0.419 \\
\hline 6 & -1 & 13 & 2 & 0.224 \\
\hline 7 & -2 & 13 & 2 & 0.273 \\
\hline 8 & -2 & -13 & 2 & 0.083 \\
\hline
\end{tabular}
The program is in G-Fortran, the calculation was performed on PC Pentium $(3.5 \mathrm{GHz})$ with the double accuracy. In table 1 gives the maximum relative error in percent

Table 1. The maximum relative error $\varepsilon$ at the solution of the telegraph equation

where $T$ - the exact explicit solution (66), $\widetilde{T}-$ an approximate analytical solution for intensive mathematical technology article for various values $k, A_{1}, C_{2}$. As can be seen from table 1 precision analytical solutions obtained in this article is satisfactory.

At compared with the numerical solution of (62) - (64) used the technology of calculating the linear telegraph equation in [18]. For the numerical computation applied non-explicit unconditionally stable difference scheme with an absolute error of approximation for the first and second derivative with respect to space $-\mathrm{O}\left[\sum_{i=1}^{3}\left(\Delta x_{i}\right)^{2}\right]$ and the three-level scheme for the time derivative with approximation error $-\mathrm{O}\left[(\Delta t)^{2}\right]$. For reference input values and $m=A_{1}=0, A_{3}=1300, t_{0}=0.2 \mathrm{~s}$, $L_{i}=0.1 \mathrm{~m}, i=1,2,3, C_{2}=1.3$ on equations (19), (27), $(45)-(47),(55)-(61)$ get $\varepsilon=9.1 \%$, and on the difference schemes [18] at $N_{i}=41, i=1,2,3, \Delta t=10^{-3}$ get $\varepsilon=$ $18.6 \%$ and $t_{c}=3 \mathrm{c}$. A specific example is the accuracy of analytical formulas (19), (27), (45) - (47), (55) - (61) was found in two times better than the accuracy of the numerical solutions [18].

In table 2 shows the solution of the wave equation by formulas (19), (27), (45) - (47), (57), (59) at different values of $m, t_{0}, L_{i}=a, i=1,2,3$. As can be seen from table 2 in the solution of the wave equation satisfactory accuracy $(\varepsilon<$ $10 \%$ ) is achieved for a small times $5 \times 10^{-6} \leq t_{0} \leq 10^{-3}$ S.

Table 2. The maximum relative error $\varepsilon$ at the solution of the wave equation

\begin{tabular}{c|c|c|r|c}
\hline № & $t_{0}$ & $\mathrm{~m}$ & $\mathrm{a}$ & $\varepsilon, \%$ \\
\hline 1 & $5 \times 10^{-6}$ & 0.5 & 0.1 & 4.29 \\
2 & $10^{-4}$ & 0 & 0.1 & 5.03 \\
3 & $5 \times 10^{-5}$ & 0.5 & 1 & 4.28 \\
4 & $10^{-3}$ & 0 & 1 & 4.96
\end{tabular}

Now, compare the accuracy of the analytical formulas (19), (27), (45) - (47), (55) in the one-dimensional case in the absence of iterations for simplified boundary value problem

$$
\begin{gathered}
z^{-1} \frac{\partial^{2} T}{\partial t^{2}}+\chi^{-1} \frac{\partial T}{\partial t}=\frac{\partial^{2} T}{\partial y^{2}}, 0<t \leq t_{0} \\
\left.T\right|_{t=0}=T_{\mathrm{н}},\left.\frac{\partial T}{\partial t}\right|_{t=0}=0,\left.T\right|_{y=0}=T_{w},\left.T\right|_{y \rightarrow \infty}=T_{\mathrm{н}}
\end{gathered}
$$

with known analytical solution of [5] at $z=c^{2}$, $\chi=A_{\mathrm{H}} / C$

$$
T(y, t)=\theta\left(T_{w}-T_{\mathrm{н}}\right)+T_{\mathrm{н}}, \theta=u(\beta-\delta) \exp (-\delta)+
$$




$$
\begin{gathered}
+\delta u(\beta-\delta) \int_{0}^{\sqrt{\beta^{2}-\delta^{2}}} \frac{I_{1}(\eta) \exp \left[-\left(\eta^{2}+\delta^{2}\right)^{0.5}\right] d \eta}{\left(\eta^{2}+\delta^{2}\right)^{0.5}}, \\
\eta=\sqrt{\beta^{2}-\delta^{2}}, \beta=c^{2} t /(2 \chi), \delta=c y /(2 \chi),
\end{gathered}
$$

where $u(\beta-\delta)$ there is the unit function $(u=1$ at $u(\beta-\delta), u=0$ for $\beta<\delta), I_{1}(\eta)$ there is modified Bessel function of the first order [5]

$$
I_{1}=\sum_{k=0}^{\infty} \frac{(\eta / 2)^{2 k+1}}{(k+1)(k !)^{2}} .
$$

As can be seen from (70) the row converge slowly at $\eta>20$. Therefore, by (69), (70), for example, in metals: steel $-c=5700 \mathrm{~m} / \mathrm{s}, \chi=5 \times 10^{-6} \mathrm{~m}^{2} / \mathrm{s}$, copper $-c=$ $4700 \mathrm{~m} / \mathrm{s}, \quad \chi=1.16 \times 10^{-4} \mathrm{~m}^{2} / \mathrm{s}$, liquid: water $-c=$ $1500 \mathrm{~m} / \mathrm{s}, \quad \chi=1.43 \times 10^{-7} \mathrm{~m}^{2} / \mathrm{s}$ [16], air and other under normal conditions, you can actually study only rapid processes $\left(t \leq 10^{-8} \mathrm{~s}\right)$ on micro distance $\left(L_{1} \leq 10^{-6} \mathrm{~m}\right)$, described by the one-dimensional problem of the form (67) and (68).

$$
\begin{aligned}
& \text { At } \chi=2 \times 10^{-5} \mathrm{~m}^{2} / \mathrm{s}, c=340 \mathrm{~m} / \mathrm{s}, T_{w}=800 \mathrm{~K}, \\
& T_{\text {н }}=293 \mathrm{~K}, L_{1}=4 \times 10^{-7} \mathrm{~m}, \quad t_{0}=2.5 \times 10^{-9} \mathrm{~s}, \beta= \\
& 7.225, \quad 0 \leq \delta \leq 3.4 \quad, \quad 0 \leq \eta \leq 6.375
\end{aligned}
$$

$\Delta \eta=6.375 /\left(N_{1}-1\right), N_{1}=51$ unlike the analytical solution (19), (27), (45) - (47), (55) in the one case the exact (69) is not exceeded $5.6 \%$.

\section{Conclusion}

1. On the basis of the locally one-dimensional scheme splitting, quasi-linearization and Laplace integral transform find an approximate analytical solution of a nonlinear hyperbolic equation of the second order, without using the theory of the series [17]. 2. In the one case, a comparison of the accuracy of analytical formulas articles with known exact solution of the telegraph type [5]. 3. The method of the trial function given the comparison of analytical solutions, the developed technology, with the exact solution of the boundary value problem with the numerical solution by a known method. 4. At the test calculations of (62) - (64) are considered conventional finite space-time intervals: $0.1 \leq L_{i} \leq 1 \quad \mathrm{~m}, \quad \mathrm{i}=1,2,3,10^{-5} \leq t_{0} \leq 0.2 \quad \mathrm{~s}$, encountered in practice $[1,3,13,15]$.

The work was supported by the Single Order Attire 1.19.09.

\section{REFERENCES}

[1] Lykov A.V. Heat and mass transfer, Handbook,Energy,Moscow 1971.

[2] Zhou D., Casas BaskesX., LebonDj. Extended irreversible thermodynamics, NITs "Regular and chaotic thermodynamics"; Instcomp. tests were performed,Moscow-Izhevsk 2006.

[3] Tichonov A.N., Samarsky A.A. The equations of mathematical physics,Science, Moscow1977.

[4] Lykov A.V. Methods for solving nonlinear transient heat conduction equations, News Academy of Sciences of the USSR, Energy and transport,Moscow,№ 5, 109-150, 1970.

[5] Baumeister K., Hamill T. Hyperbolic equation of heat conduction. The solution of the semi-infinite body, Heattransfer,№ 4, 112-119, 1969.

[6] Kudinov V.A., Kudinov I.V. Preparation and analysis of exact analytical solutions of hyperbolic-parameter equation of heat conduction for a flat wall,Thermal physics of high temperature,Moscow,vol. 50, № 1,118-125, 2012.

[7] Yakimov A.S. The analytical method for solving boundary value problems, Ed. 2-nd, add, Publishing House of Tomsk. University Press, Tomsk 2011.

[8] Bellman R., Kalaba R. Quasi-linearization and nonlinear boundary value problems, Springer-Verlag, New York 1968.

[9] Kantorovich L.V. Functional analysis and applied mathematics,Success of mathematical sciences,Moscow,№ 6, 89-185, 1948.

[10] Samarsky A.A. On numerical methods for solving mathematical physics, Heat and mass transfer, Science and technology, Minsk, vol. 11, 990-1006, 1969.

[11] Samarsky A.A. Introduction to the theory of difference schemes,Science, Moscow1971.

[12] Ditkin V.A., Prudnikov A.P. Operational calculus, High school, Moscow 1966.

[13] Doetsch G. Guide to the practical application of the Laplace transform, Physical and mathematical State edition, Moscow 1960.

[14] Matveev N.M. Methods for the integration of ordinary differential equations, High school, Moscow 1967.

[15] Yakimov A.S. On a method of solving the conjugate heat transfer problem. Part 2, Journal of Engineering Physics, Minsk, vol. 86, № 3, 464-474, 2013.

[16] Koshkin N.I., Shirkevich M.G. Handbook of elementary physics, Science, Home edition of the physical and mathematical literature, Moscow 1988.

[17] Bronstein N.I., Semendyaev L.A. Handbook of mathematics for engineers and students of VTU-call,Science, Home edition of thephysical and mathematical literature, Moscow 1986.

[18] Grishin A.M., Yakimov A.S. The iterative-interpolation method for solving three-dimensional wave equation, Computational technologies, Novosibirsk, vol. 12, № 1, 22-34, 2007. 